\title{
Avaliação da Performance de Estratégias de Decisão de Mobilidade MADM na Perspectiva da Eficiência de QoE
}

\author{
Mathews P. S. Lima ${ }^{1}$, Charles H. F. dos Santos ${ }^{1}$, Felipe S. Dantas Silva ${ }^{1,2}$ \\ Augusto Venâncio Neto ${ }^{2,3}$ \\ ${ }^{1}$ Laboratório de Tecnologias Avançadas em Redes de Computadores (LaTARC) \\ Instituto Federal de Educação, Ciência e Tecnologia do Rio Grande do Norte (IFRN) \\ Natal-RN, Brasil \\ ${ }^{2}$ Departamento de Informática e Matemática Aplicada (DIMAp) \\ Universidade Federal do Rio Grande do Norte (UFRN) - Natal-RN, Brasil \\ mathews.phillippeacademico.ifrn.edu.br
\{charles.hallan, felipe.dantas\}@ifrn.edu.br, augusto@dimap.ufrn.br
}

\begin{abstract}
The state of the art in MADM quality-oriented mobility management evaluation only address network-/packet-level Quality of Service (QoS) measures. Therefore, this work fills this gap through a broad review of the MADM methods applied to the quality-oriented mobility decision process, as well as a user-centric Quality of Experience (QoE) assessment of these algorithms about our previous work: the Extended Elitism for Best Selection (E2BS). The QoE evaluation is conducted leveraging the flexibility of the Software-Defined Mobile Networking (SDMN) paradigm. The QoE analysis outcomes demonstrate that E2BS outperforms the selected MADM-based methods, by improving the overall QoE perceived by mobile users when consuming multimedia mobile flows.
\end{abstract}

Resumo. O estado da arte na avaliação de gerenciamento de mobilidade orientada para a qualidade por meio de técnicas de decisão MADM apenas aborda as medidas dos níveis de Qualidade de Serviço (Quality of Service - QoS) dos pacotes das redes. Nesse sentido, este trabalho preenche esta lacuna através de uma ampla revisão dos métodos MADM aplicados ao processo de decisão de mobilidade orientada para a qualidade por meio da realização de uma avaliação de QoE, centrada no usuário, desses algoritmos em relação ao nosso trabalho anterior: o Extended Elitism for Best Selection (E2BS). A avaliação de QoE é conduzida por meio da flexibilidade provida pelo paradigma de Redes Móveis Definidas por Software (Software-Defined Mobile Networking-SDMN). Os resultados da análise QoE demonstram que o E2BS supera os métodos selecionados baseados em MADM, melhorando a QoE percebida pelos usuários móveis ao consumir fluxos móveis multimídia.

\section{Introdução}

Com o surgimento de várias tecnologias de redes móveis, tal como o IEEE 802.11 [Group et al. 1999], 3GPP 3G/4G, 5G [Gupta and Jha 2015] e dos objetos móveis (Moving Objects - MO) (e.g. laptops, smartphones, drones etc.) que atuam como nós de rede autônomos com diferentes padrões de mobilidade, ganharam notoriedade as arquiteturas de Redes sem Fio para Objetos Móveis (Wireless Network for Moving Objects 
- WiNeMO) [Silva et al. 2016] que viabilizam a implantação de infraestruturas com capacidade de oferecer uma gama de novos serviços e aplicações, tais como mobilidade, Qualidade de Serviço (Quality of Service - QoS), dentre outros. Esse cenário também levou à convergência de várias aplicações com requisitos críticos de QoS (e.g. largura de banda mínima, tolerância a atraso, perda de pacotes e variação do atraso etc.), como Vídeo sob Demanda (Video on-Demand - VoD) e Voz sobre IP (Voice over IP - VoIP), que exigem novas estratégias de gerenciamento das infraestruturas que sejam capazes de fornecer garantias de comunicações móveis altamente confiáveis.

Estimativas recentes ${ }^{1}$ apontaram que 429 milhões de novos dispositivos móveis foram introduzidos na Internet em 2016, o que causou um acréscimo na taxa média mensal do tráfego móvel em 63\% em relação a 2015 (i.e. 7.2 Exabytes comparados com 4.4). A expectativa atual é que esses valores possam atingir 49 Exabytes em 2021, o que representa um crescimento de aproximadamente $581 \%$ em relação a 2016 . Este crescimento significativo no tráfego de dados móveis é um dos muitos reflexos da evolução das aplicações multimídia consumidas a partir de dispositivos móveis nos últimos anos. Uma vez que essas aplicações exigem alta largura de banda, o tráfego multimídia tem sido caracterizado como um dos principais responsáveis pela criação de gargalos nas redes.

Esse crescimento da demanda por conteúdo multimídia e serviços em tempo real exigem que as redes móveis forneçam mecanismos avançados de controle capazes de suportar diferentes requisitos de tráfego, ao mesmo tempo em que oferecem diferentes níveis de qualidade. Isto aplica-se especialmente ao processo de handover [Zekri et al. 2012], em que a infraestrutura seja capaz de viabilizar a continuidade da conexão dos MOs através da seleção de uma rede, dentre as redes adjacentes em sua área de cobertura, com melhores condições durante períodos de mobilidade com o intuito de mantê-los sempre melhor conectados.

Para alcançar esse objetivo, o mecanismo de decisão de handover deve implementar procedimentos orientados para a qualidade, o que significa a realização desse processo levando em consideração os requisitos de qualidade mínima do(s) fluxo(s) da sessão móvel combinados com as condições atuais da conexão sem fio em relação aos PoAs candidatos.

A literatura relacionada revela vários casos de uso envolvendo a adoção de esquemas de decisão de handover orientados para a qualidade nos sistemas de gerenciamento de mobilidade. Dentre estes, os Métodos de Decisão Multiatributo (Multiple Attribute Decision Making - MADM) [Yoon et al. 1995] são considerados como as estratégias que possuem maior confiabilidade devido à sua flexibilidade e precisão na seleção de redes móveis que melhor atendam as necessidades dos MOs [Zekri et al. 2012].

Embora os sistemas WiNeMO ofereçam a perspectiva de proporcionar conectividade ubíqua e orientada para a qualidade [Silva et al. 2016], os seus mecanismos de gerência de mobilidade devem garantir a seleção das melhores redes que considerem os requisitos de QoS dos MOs para assegurar níveis aceitáveis de Qualidade de Experiência (Quality of Experience - QoE), mesmo durante os períodos de handover, de forma dinâmica e transparente [Golmie 2009].

\footnotetext{
${ }^{1}$ https://www.cisco.com/c/en/us/solutions/collateral/service-provider/visual-networking-indexvni/mobile-white-paper-c11-520862.html
} 
Estudos anteriores [Martinez-Morales et al. 2010] realizaram avaliações em larga escala de métodos MADM. No entanto, são considerados insuficientes por realizarem apenas análise estatística da rede selecionada e dos atributos de QoS, o que não assegura que a rede selecionada seja, de fato, a melhor rede. Uma avaliação abrangente de técnicas de decisão de handover deve fornecer um conjunto extensivo de referências para auxiliar os pesquisadores a escolherem a estratégia mais apropriada para um mecanismo particular de controle de mobilidade, especialmente quando trata-se de fluxos de sessões de aplicações multimídia em redes móveis. Assim sendo, empregar um mecanismo ineficiente limitaria sua operacionalidade e restringiria rigorosamente seu escopo em cenários WiNeMO.

Diante disso, este trabalho provê uma ampla avaliação dos principais métodos MADM aplicados ao campo de decisão de mobilidade orientado para a qualidade através de uma minuciosa revisão a respeito de suas características, além de realizar uma avaliação dos efeitos das decisões realizadas por cada método sob a perspectiva de fluxos de aplicações multimídia móvel.

As avaliações foram conduzidas a partir de implementações dos principais métodos (17 no total) de decisão de mobilidade baseados em MADM para operar sob uma infraestrutura de Redes Móveis Definidas por Software (Software-Defined Mobile Networking - SDMN) [Liyanage et al. 2015], virtualizada no emulador Mininet-Wifi [Fontes et al. 2015], capaz de reproduzir condições IEEE 802.11g reais. A abordagem oferecida pelo paradigma SDMN viabilizou a extensão do plano de controle capacitandoo a realizar decisão de mobilidade orientadas para a qualidade a fim de melhor explorar as perspectivas de operação dos mecanismos decisórios de cada método considerado em meio a um cenário de antecipação de handover com perspectivas de execução transparente.

Outra contribuição deste estudo é a inclusão do método MADM Extended Elitism for Best Selection (E2BS), fruto de pesquisas anteriores de nosso grupo de pesquisa [Silva et al. 2014], nesta avaliação. Os resultados demonstraram que o E2BS supera os demais fornecendo PoAs mais adequados aos fluxos de sessões multimídia móveis sendo o método MADM mais apropriado para adoção em cenários de mobilidade envolvendo este tipo de tráfego.

O restante deste trabalho está organizado da seguinte maneira: a Seção 2 aponta os trabalhos relacionados ao tema de pesquisa descrito, de modo a delinear as contribuições desta proposta; a Seção 3 descreve a metodologia utilizada na avaliação e uma discussão dos resultados; a Seção 4 apresenta as considerações finais e aponta direções para trabalhos futuros.

\section{Trabalhos Relacionados}

Inúmeros trabalhos publicados na literatura tratam da avaliação dos mecanismos de decisão de mobilidade baseadas em MADM, no entanto, a análise da percepção do usuário é ignorada frequentemente. Destes trabalhos, a maioria apenas realizou análises estatísticas e seus resultados foram apresentados em termos de parâmetros de rede (analisando apenas os parâmetros de QoS dos PoAs selecionados, como vazão, perda de pacotes, atraso e jitter etc.). A Tabela 1 apresenta os principais estudos que realizaram revisões de métodos baseados em MADM, avaliando os trabalhos disponíveis nos seguintes termos, 
de acordo com uma revisão levantada em um trabalho anterior do nosso grupo de pesquisa [dos Santos et al. 2017]: (i) se o trabalho realizou avaliações em termos de QoE e; (ii) número de métodos MADM revisados.

\begin{tabular}{ccc}
\hline Trabalho & Avaliação QoE & No de Métodos $^{\text {Nõa }}$ \\
\hline [Lahby et al. 2015] & Não & 4 \\
[Tran et al. 2008] & Não & 1 \\
[Stevens-Navarro et al. 2006] & Não & 4 \\
[Morales et al. 2010] & Não & 7 \\
[Mohamed et al. 2012] & Não & 3 \\
[Drissi et al. 2015] & Não & 3 \\
[Obayiuwana and Falowo 2017] & Não & 11 \\
[dos Santos et al. 2017] & Sim & 5 \\
Proposta Atual & Sim & $\mathbf{1 7}$ \\
\hline
\end{tabular}

Tabela 1. Revisão dos trabalhos relacionados

Desta maneira, até onde sabemos, e comprovado pelos estudos aprofundados na literatura disponível, nenhum outro trabalho apresentou uma revisão abrangente sobre os principais métodos de decisão de mobilidade orientada para a qualidade baseada em MADM, incluindo avaliações sob a perspectiva de QoE. Com base nisso, a proposta apresentada neste trabalho estende as perspectivas fornecidas em nosso trabalho anterior [dos Santos et al. 2017] por meio de uma ampla avaliação dos métodos MADM aplicados ao gerenciamento de mobilidade documentados na literatura.

\section{Avaliação dos métodos de decisão baseado em MADM}

A avaliação de eficiência dos algoritmos MADM foi conduzida sob a abordagem de Gerenciamento de Mobilidade Infraestruturada (Infrastructured Mobility Management - IMM) [Silva et al. 2016] materializada por meio da flexibilidade oferecida pelo paradigma de redes SDMN, conforme o testbed mostrado na Figura 1(a).

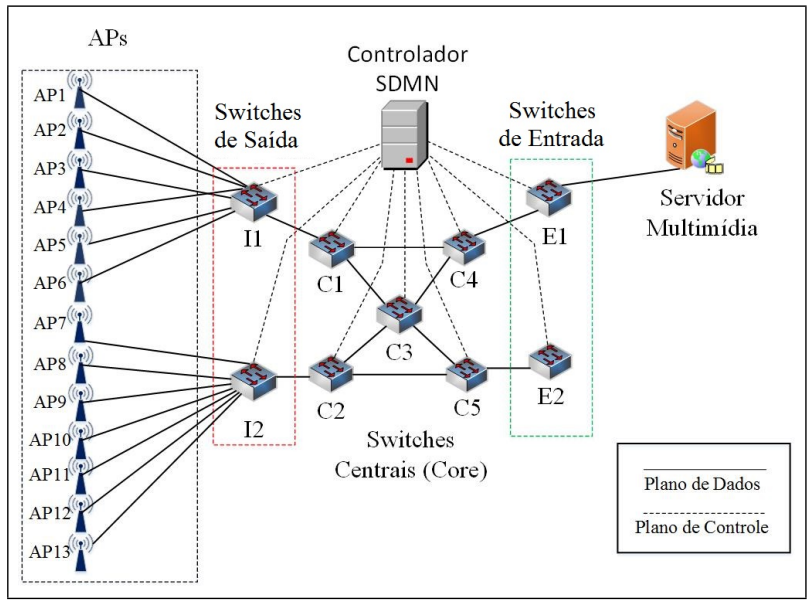

(a)

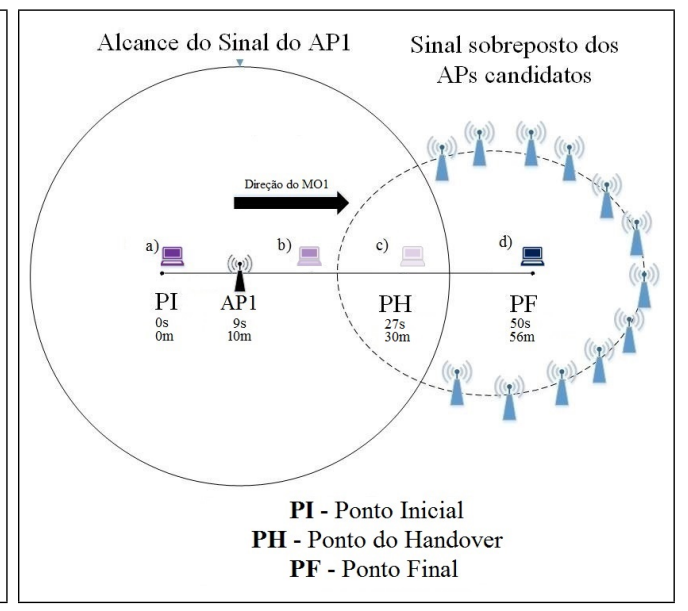

(b)

Figura 1. Testbed e Representação da Mobilidade realizada pelo MO

O testbed foi configurado para habilitar uma infraestrutura SDMN, composto por 9 switches OpenFlow [McKeown et al. 2008] e uma infraestrutura de rede sem fio com 
13 PoAs, ambos baseados no Open vSwitch. O plano de controle da infraestrutura SDMN ficou a cargo do Controlador SDN Ryu ${ }^{2}$, configurado em um host desktop HP Compaq 6005, AMD Phenom(TM) II X4, 8G RAM, 500 GB de disco rígido e Sistema Operacional Linux Ubuntu 16.04 LTS.

Toda a implementação das funcionalidades do Controlador SDMN levou em consideração as funcionalidades necessárias para o provimento de um serviço de gerenciamento de mobilidade orientada para a qualidade. Para habilitar mobilidade através da abordagem soft-handover, foi implementado no plano de controle SDMN um esquema de antecipação de mobilidade baseado no sistema SDWiNeMO [Silva et al. 2016], que leva em consideração limites predefinidos de parâmetros de rede (e.g. limite do RSSI para detectar quando um MO está prestes a perder conectividade com o PoA atual). O OpenFlow é usado para aplicar funções de rede tanto nos elementos da infraestrutura cabeada quanto da sem fio, permitindo que os elementos de rede possam ser configurados remotamente, ao passo que os aspectos de mobilidade dos MOs (e.g. varredura de rede, obtenção de parâmetros estatísticos do meio sem fio, configuração de handover etc.) são conduzidos sob uma abordagem baseada no padrão IEEE 802.21 (Media Independent Handover MIH) [Golmie et al. 2009].

\begin{tabular}{cccc}
\hline $\begin{array}{c}\text { Método } \\
\text { MADM }\end{array}$ & $\begin{array}{c}\text { PoA } \\
\text { Selecionado }\end{array}$ & $\begin{array}{c}\text { VQM } \\
\text { Pontuação Média }\end{array}$ & $\begin{array}{c}\text { SSIM } \\
\text { Pontuação Média }\end{array}$ \\
\hline TOPSIS & AP3 & 3.1266 & 0.9094 \\
MEW & AP12 & 1.9864 & 0.9490 \\
SAW & AP9 & 2.1476 & 0.9483 \\
GRA & AP12 & 1.9090 & 0.9405 \\
E2BS & AP5 & 1.8757 & 0.9548 \\
ELECTRE & AP2 & 3.5037 & 0.8617 \\
VIKOR & AP10 & 2.6153 & 0.9272 \\
DiA & AP13 & 2.7923 & 0.9210 \\
PROMETHEE & AP12 & 1.9659 & 0.9520 \\
MULTIMOORA & AP12 & 2.1962 & 0.9420 \\
M-SAW & AP6 & 3.0530 & 0.9114 \\
TOPSIS-based \#1 & AP8 & 3.2975 & 0.8843 \\
TOPSIS-based \#2 & AP11 & 3.2100 & 0.8996 \\
TOPSIS-based \#3 & AP11 & 3.0163 & 0.9098 \\
GRA-based \#1 & AP4 & 3.4491 & 0.9003 \\
GRA-based \#2 & AP12 & 1.9283 & 0.9541 \\
GRA-based \#3 & AP7 & 1.9140 & 0.9515 \\
\hline
\end{tabular}

Tabela 2. Pontuação média de QoE nos PoAs selecionados por cada algoritmo

Nessa avaliação, o host MO1 move-se de uma posição inicial (Ponto Inicial - PI), onde tem uma conexão estabelecida com o PoA AP1, conforme mostra a Figura 1(b), em direção posição final (Ponto Final - PF) com 12 PoAs com sinais sobrepostos e com valores variados para os atributos de QoS. No meio do percurso (Ponto de Handover PH), o MO1 perde a conexão com o AP1, e será submetido ao processo de soft-handover. Ao final do processo de mobilidade, a análise de QoE é realizada. Essa análise é baseada na comparação entre o vídeo de referência, transmitido pelo servidor multimídia, e o vídeo recebido pelo MO1.

\footnotetext{
${ }^{2}$ https://osrg.github.io/ryu
} 
Os pesos de cada atributo, foram definidos com base nos cálculos do Analytical Hierarchy Process (AHP) [Lahby et al. 2013], que levam em consideração os requisitos de QoS de aplicações de vídeo em tempo real (Access Category Video - AC_VI), conforme o padrão IEEE 802.11e [IEEE Std 802.11e-2005 2005].

A mídia do vídeo, entregue pelo Servidor Multimídia (Figura 1(a)), consiste em um vídeo real enviado através da ferramenta Evalvid [Klaue et al. 2003], contendo as seguintes características: codec H.264; resolução 1920x1080 (HDTV 1080p); taxa de 25 quadros por segundo Frames per Second - FPS); taxa de bits de 3 Mbps e duração de 47 segundos com 1177 quadros. Com o intuito de reproduzir um cenário com alta demanda de recursos, foram definidas 102 sessões multimídia adicionais, sendo uma para cada MO, distribuídas (de forma randômica) entre os PoAs da infraestrutura SDMN.

Cada MO adicional, distribuído no testbed, está associado a uma sessão multimídia distinta e com exigências de QoS diferente, como taxa de bits mínima. Para este fim, a ferramenta Iperf foi utilizada para gerar tráfego sintético para cada uma das 102 sessões multimídia adicionais.

Enquanto é feita a avaliação da eficiência da decisão de cada método durante a transmissão do conteúdo multimídia, a medida de qualidade da imagem é realizada para calcular uma pontuação que represente os níveis de QoE. Por meio das métricas de QoE VQM e SSIM é possível avaliar o impacto do comportamento de cada método na seleção de uma nova rede móvel. As medidas foram obtidas usando a ferramenta MSU Video Quality Measurement Tool (VQMT ${ }^{3}$ ), que utiliza como entrada ambos os arquivos dos vídeos: o original (enviado pelo Servidor Multimídia) e o processado (recebido pelo MO1). A Tabela 2 apresenta um resumo das pontuações médias totais obtidas por cada método MADM utilizando VQM e pelo SSIM durante os experimentos.

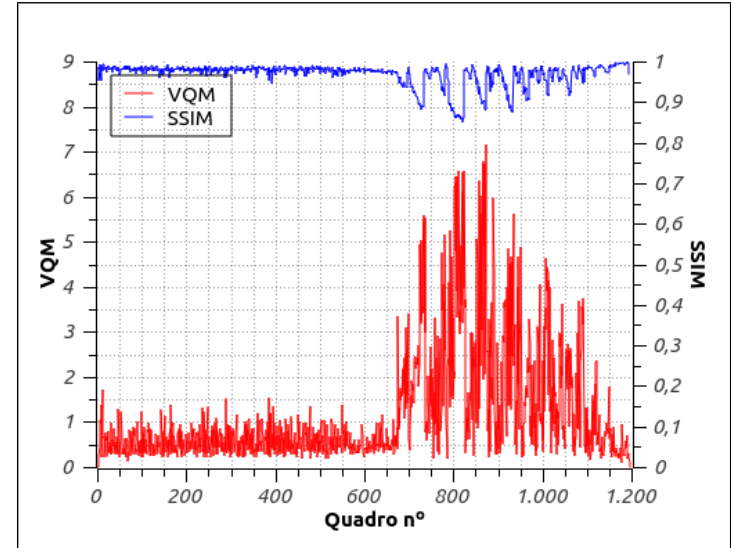

(a) E2BS

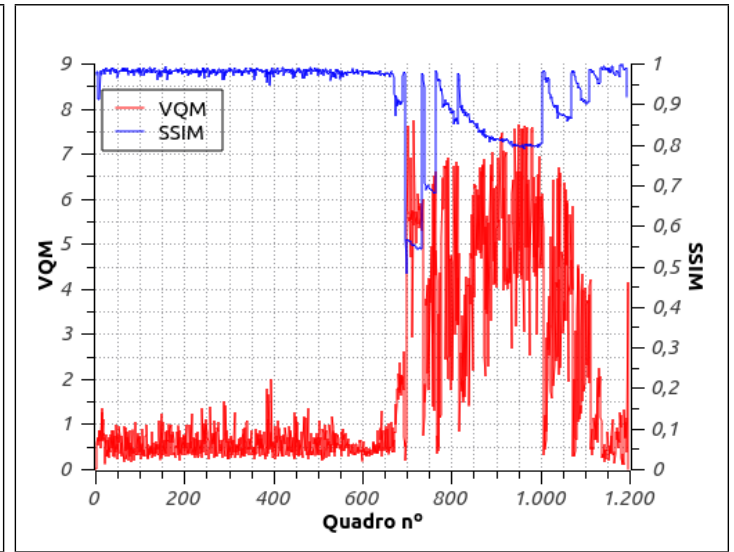

(b) ELECTRE

Figura 2. Resultados do E2BS

O algoritmo de decisão E2BS selecionou a rede AP5 dentre as redes candidatas, que apresenta o melhor desempenho nas métricas VQM e SSIM entre todos os algoritmos. Em relação ao pior caso (ELECTRE), a melhora entre os valores de VQM e SSIM são de $86 \%$ e $11 \%$, respectivamente. Os gráficos das Figuras 2(a) e 2(b) mostram o desempenho das rede escolhida pelos algoritmos E2BS e ELECTRE.

\footnotetext{
${ }^{3} \mathrm{http}: / /$ compression.ru/video/quality_measure
} 


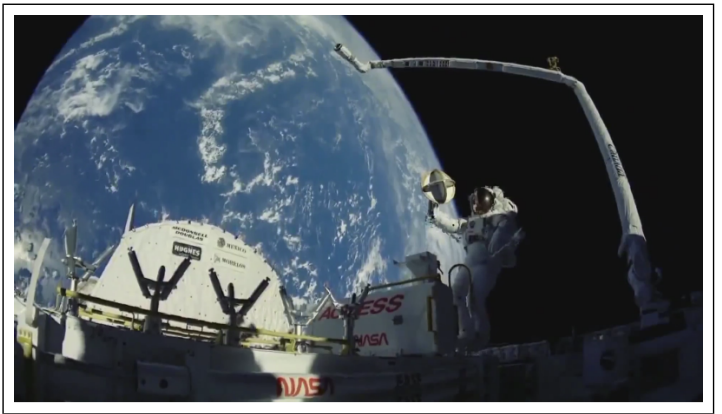

(a) E2BS

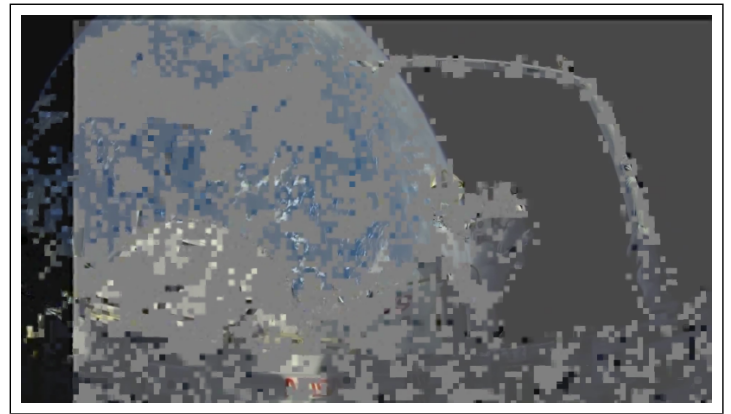

(b) ELECTRE

Figura 3. Comparação de quadros das sessões providas pelas decisões do E2BS e ELECTRE

A Figura 3 mostra a perspectiva do usuário sobre o conteúdo recebido a partir da extração de um quadro aleatório $\left(\mathrm{n}^{\mathrm{0}} 727\right)$ em um momento da transmissão após o handover para a nova rede. Este resultado, que apresenta o melhor (E2BS) e o pior (ELECTRE) nas Figuras 3(a) e 3(b), respectivamente, de todo o conjunto de avaliações confirmam os resultados providos pelas métricas de QoE.

\section{Conclusão}

Este trabalho apresentou uma revisão das principais contribuições na área de mobilidade que contemplam os mecanismos de decisão de mobilidade propostos na literatura a partir do uso de técnicas baseadas em SDMN, contribuindo ainda com a demonstração dos efeitos da decisão de cada um dos métodos MADM sobre a qualidade multimídia percebida pelo usuário móvel. Através de estudos aprofundados de trabalhos relacionados, não há indícios de trabalhos anteriores que tenham se comprometido em apresentar uma ampla revisão de métodos MADM aplicados ao processo de gerenciamento de mobilidade orientada à qualidade, bem como uma avaliação dos resultados das decisões de cada método sob a perspectiva de QoE.

Os resultados revelaram que o E2BS obteve um maior grau de precisão na seleção de uma rede melhor, levando em consideração os requisitos de qualidade da sessão multimídia móvel. Em um estudo futuro também realizaremos um novo conjunto de análises do comportamento E2BS no que diz respeito ao custo efetivo da decisão e ao fenômeno de reversão de classificação.

\section{Referências}

dos Santos, C. H., de Lima, M. P., Silva, F. S. D., and Neto, A. (2017). Performance evaluation of multiple attribute mobility decision models: A qoe-efficiency perspective. In 2017 IEEE 13th International Conference on Wireless and Mobile Computing, Networking and Communications (WiMob), pages 159-166. IEEE.

Fontes, R., Afzal, S., Brito, S., Santos, M., and Rothenberg, C. (2015). Mininet-WiFi: Emulating software-defined wireless networks. In 2015 11th International Conference on Network and Service Management (CNSM), pages pp. 384-389.

Golmie, N. (2009). Seamless mobility: are we there yet? IEEE Wireless Communications, 16(4):12-13. 
Golmie, N., Melia, T., Bajko, G., Das, S., and Zúñiga, J.-C. (2009). IEEE 802.21 Mobility Services Framework Design (MSFD). RFC 5677.

Group, I. . W. et al. (1999). Part11: Wireless lan medium access control (mac) and physical layer (phy) specifications. ANSI/IEEE Std. 802.11.

Gupta, A. and Jha, R. K. (2015). A survey of 5g network: Architecture and emerging technologies. IEEE Access, 3:1206-1232.

IEEE Std 802.11e-2005 (2005). IEEE standard for information technology-local and metropolitan area networks-specific requirements-part 11: Wireless lan medium access control (MAC) and physical layer (PHY) specifications - amendment 8: Medium access control (mac) quality of service enhancements. IEEE Std 802.11e (Amendment to IEEE Std 802.11, 1999 Edition (Reaff 2003), pages 1-212.

Klaue, J., Rathke, B., and Wolisz, A. (2003). Evalvid - a framework for video transmission and quality evaluation. In 13th Int. Conference on Modelling Techniques and Tools (TOOLS 2003), pages pp. 255-272.

Lahby, M., Cherkaoui, L., and Adib, A. (2013). A novel ranking algorithm based network selection for heterogeneous wireless access. $J N W, 8(2): 263-272$.

Liyanage, M., Gurtov, A., and Ylianttila, M. (2015). SDMN, pages 440-. Wiley Telecom.

Martinez-Morales, J. D., Pineda-Rico, U., and Stevens-Navarro, E. (2010). Performance comparison between madm algorithms for vertical handoff in $4 \mathrm{~g}$ networks. In Electrical Engineering Computing Science and Automatic Control (CCE), 2010, pages 309314. IEEE.

McKeown, N., Anderson, T., Balakrishnan, H., Parulkar, G., Peterson, L., Rexford, J., Shenker, S., and Turner, J. (2008). OpenFlow: enabling innovation in campus networks. SIGCOMM Comput. Commun. Rev., vol. 38(2):pp. 69-74.

Obayiuwana, E. and Falowo, O. E. (2017). Network selection in heterogeneous wireless networks using multi-criteria decision-making algorithms: a review. Wireless Networks, 23(8):2617-2649.

Silva, F., Castillo-Lema, J., Neto, A., Silva, F., Rosa, P., Corujo, D., Guimarães, C., and Aguiar, R. (2014). Entity title architecture extensions towards advanced qualityoriented mobility control capabilities. In Computers and Communication (ISCC), 2014 IEEE Symposium on, pages 1-6. IEEE.

Silva, F. S. D., Neto, A. V., Maciel, D., Castillo-Lema, J., Silva, F., Frosi, P., and Cerqueira, E. (2016). An innovative software-defined winemo architecture for advanced qos-guaranteed mobile service transport. Computer Networks, 107:270-291.

Yoon, P. K., Hwang, C.-L., and Yoon, K. (1995). Multiple Attribute Decision Making: An Introduction (Quantitative Applications in the Social Sciences). Sage Pubn Inc.

Zekri, M., Jouaber, B., and Zeghlache, D. (2012). A review on mobility management and vertical handover solutions over heterogeneous wireless networks. Computer Communications, 35(17):2055-2068. 